\author{
IDENTIFICATION AND CHARACTERIZATION OF \\ CONSERVATIVE ORganic Tracers FOR USE AS \\ HYDROLOGIC TRACERS FOR THE YUCCA MOUNTAIN \\ Site Characterization STUdY
}

PROGRESS REPORT

JULY 1, 1995 to SEPTEMBER 30, 1995

DOE Cooperative Agreement

No. DE-FC 08-90NV10872

\author{
Klaus Stetzenbach \\ Irene Farnham
}
Harry Reid Center For Environmental Studies University of Nevada - Iras Vegas

\title{
DISCLAIMER
}

This report was prepared as an account of work sponsored by an agency of the United States Government. Neither the United States Government nor any agency thereof, nor any of their employees, makes any warranty, express or implied, or assumes any legal liability or responsibility for the accuracy, completeness, or usefulness of any information, apparatus, product, or process disclosed, or represents that its use would not infringe privately owned rights. Reference herein to any specific commercial product, process, or service by trade name, trademark, manufacturer, or otherwise does not necessarily constitute or imply its endorsement, recommendation, or favoring by the United States Government or any agency thereof. The views and opinions of authors expressed herein do not necessarily state or reflect those of the United States Government or any agency thereof. 
The laboratory work to support the selection of the tracers to be used for the C-Well tracer tests has been completed. The solubilities for each of the fluorinated benzoic acids in J13 water have been determined and the stability of these compounds to both degradation and sorption on ground tuff has been measured through batch and column testing. Method development work is in progress for the analysis of low levels of 2,6-difluorobenzoic acid. The current plan is to use this compound as a tracer in a preliminary test.

\section{BATCH TESTING}

The batch tests provide information on the stabilities of the potential tracers in an environment that closely simulates that of the $\mathrm{C}$ Wells. Mixtures containing these compounds in $\mathrm{J}-13$ water are exposed to three types of tuffs (light, medium, and dark). The tuff material has been identified by DOE geologists as Bullfrog Tuff, a crystal rich, pumiceous, rhyolitic (silica rich) tuff which underlies the Topopah Spring tuff in Yucca Mountain. The samples referred to as light, medium and dark have been classified as follows: Un-welded "light" tuff, which is light weight, porous, and easily broken; Moderately-welded "medium" tuff, which is semi-porous, and contains some dense areas of collapsed pumice fragments; and the Densely-welded "dark" tuff which is hard, very dense and vitrophyric in nature, and can be classified as an obsidian.

A control is also prepared which contains each of the tracers in J-13 water but no tuff. The concentration of each compound is measured periodically to determine changes that occur with time. High performance liquid chromatography (HPLC) is used to separate each of the compounds within the mixture and an ultraviolet (UV) or fluorescence detector is used for detection and quantitation.

All commercially available fluorinated benzoic acids have been batch tested over sixty days. These compounds exhibit excellent stability with changes in concentation of less than $5 \%$ in each tuff type for most compounds. The concentrations for each of the compounds in each of the tuff types from day 0 to day 60 are shown in the following tables. These data are also presented in the graphs in Figures 1-17. These graphs not only show the high stability of these compounds but also demonstrate the excellent precision available through the HPLC/UV methods.

Table 1 Concentrations of Tracers in the Control Samples

\begin{tabular}{|c|c|c|c|c|c|c|c|}
\hline \multirow[b]{2}{*}{ Compound } & \multicolumn{7}{|c|}{ Concentration (ppm) } \\
\hline & 0 & 1 & 5 & 10 & 20 & 30 & 60 \\
\hline 2,3-Difluorobenzoic Acid & 5.15 & 5.19 & 5.09 & 5.11 & 5.13 & 5.21 & 5.12 \\
\hline 2,4-Difluorobenzoic Acid & 5.33 & 5.32 & 5.35 & 5.38 & 5.25 & 5.34 & 5.37 \\
\hline 2,5-Difluorobenzoic Acid & 5.14 & 5.15 & 5.14 & 5.18 & 5.13 & 5.17 & 5.15 \\
\hline 2,6-Difluorobenzoic Acid & 4.99 & 4.97 & 5.03 & 5.01 & 5.02 & 5.17 & 5.06 \\
\hline 3,4-Difluorobenzoic Acid & 5.05 & 5.06 & 5.01 & 5.13 & 5.04 & 5.07 & 5.01 \\
\hline 3,5-Difluorobenzoic Acid & 5.06 & 5.20 & 5.01 & 5.15 & 5.05 & 5.20 & 5.03 \\
\hline 2,3,4-Trifluorobenzoic Acid & 4.95 & 4.92 & 4.92 & 5.06 & 4.90 & 5.01 & 4.98 \\
\hline 2,3,6-Trifluorobenzoic Acid & 5.10 & 5.13 & 5.04 & 5.07 & 5.06 & 5.18 & 5.13 \\
\hline 2,4,5-Trifluorobenzoic Acid & 5.92 & 5.94 & 5.94 & 6.03 & 6.08 & 6.02 & 5.90 \\
\hline 2,4,6-Trifluorobenzoic Acid & 5.74 & 5.77 & 5.79 & 5.78 & 5.84 & 5.75 & 5.72 \\
\hline 3,4,5-Trifluorobenzoic Acid & 5.14 & 5.16 & 5.02 & 5.06 & 5.31 & 5.20 & 5.06 \\
\hline 2,3,4,5-Tetrafluorobenzoic Acid & 5.74 & 5.71 & 5.66 & 5.70 & 5.73 & 5.65 & 5.60 \\
\hline 2,3,5,6-Tetrafluorobenzoic Acid & 6.20 & 6.22 & 6.29 & 6.26 & 6.21 & 6.27 & 6.24 \\
\hline Pentafluorobenzoic Acid & 5.62 & 5.64 & 5.67 & 5.71 & 5.67 & 5.65 & 5.59 \\
\hline$\alpha, \alpha, \alpha$-Trifluoro-o-toluic acid & 5.14 & 5.15 & 5.00 & 5.07 & 4.96 & 5.09 & 5.05 \\
\hline$\alpha, \alpha, \alpha-$-Trifluoro-m-toluic acid & 5.13 & 5.12 & 5.02 & 5.06 & 5.18 & 5.12 & 5.06 \\
\hline$\alpha, \alpha, \alpha$-Trifluoro-p-toluic acid & 5.28 & 5.29 & 5.19 & 5.22 & 5.23 & 5.26 & 5.14 \\
\hline
\end{tabular}


Table 2 Concentrations of Tracers in the Light Tuff Samples

\begin{tabular}{lccccccr}
\multicolumn{1}{c}{ Compound } & 0 & 1 & 5 & 10 & 20 & 30 & 60 \\
\hline 2,3-Difluorobenzoic Acid & 5.15 & 5.19 & 5.07 & 5.02 & 5.00 & 5.09 & 5.09 \\
2,4-Difluorobenzoic Acid & 5.43 & 5.45 & 5.43 & 5.35 & 5.23 & 5.35 & 5.49 \\
2,5-Difluorobenzoic Acid & 5.19 & 5.18 & 5.19 & 5.18 & 5.10 & 5.19 & 5.28 \\
2,6-Difluorobenzoic Acid & 4.77 & 4.78 & $*$ & $*$ & $*$ & $* 6.58$ & 4.96 \\
3,4-Difluorobenzoic Acid & 5.07 & 5.14 & 5.06 & 5.10 & 4.99 & 5.06 & 5.18 \\
3,5-Difluorobenzoic Acid & 5.07 & 5.08 & 5.03 & 5.04 & 5.08 & 5.11 & 5.10 \\
2,3,4-Trifluorobenzoic Acid & 4.98 & 5.04 & 5.08 & 5.11 & 4.94 & 5.06 & 5.11 \\
2,4,5-Trifluorobenzoic Acid & 5.97 & 5.99 & 5.95 & 5.97 & 6.01 & 6.02 & 6.08 \\
3,4,5-Trifluorobenzoic Acid & 5.05 & 5.08 & 5.08 & 5.13 & 5.18 & 5.25 & 5.35 \\
2,3,4,5-Tetrafluorobenzoic Acid & 5.78 & 5.81 & 5.75 & 5.69 & 5.67 & 5.65 & 5.71 \\
Pentafluorobenzoic Acid & 5.53 & 5.58 & 5.55 & 5.16 & 5.27 & 4.96 & 5.55 \\
$\alpha, \alpha, \alpha-$ Trifluoro-o-toluic acid & 4.97 & 5.02 & 4.98 & 5.03 & 4.91 & 5.06 & 5.29 \\
$\alpha, \alpha, \alpha-$ Trifluoro-m-toluic acid & 5.06 & 5.17 & 5.14 & 5.14 & 5.13 & 5.26 & 5.10 \\
$\alpha, \alpha, \alpha-$ Trifluoro-p-toluic acid & 5.16 & 5.28 & 5.25 & 5.28 & 5.23 & 5.33 & 5.31
\end{tabular}

*A large interference was observed in all of the light tuff samples. This interferred with the quantitation of $2,3,5,6-$ Tetrafluorobenzoic acid, 2,4,6-Trifluorobenzoic acid, 2,3,6-Trifluorobenzoic acid and t5 - t30 for 2,6-Difluorobenzoic acid. These data are therefore not reported.

Table 3 Concentrations of Tracers in the Medium Tuff Samples

\begin{tabular}{|c|c|c|c|c|c|c|c|}
\hline \multirow[t]{2}{*}{ Compound } & \multicolumn{7}{|c|}{ Concentration (ppm) } \\
\hline & 0 & 1 & 5 & 10 & 20 & 30 & 60 \\
\hline 2,3-Difluorobenzoic Acid & 5.17 & 5.18 & 5.11 & 5.15 & 5.11 & 5.14 & 5.10 \\
\hline 2,4-Difluorobenzoic Acid & 5.41 & 5.41 & 5.37 & 5.39 & 5.25 & 5.36 & 5.45 \\
\hline 2,5-Difluorobenzoic Acid & 5.19 & 5.18 & 5.20 & 5.18 & 5.14 & 5.21 & 5.24 \\
\hline 2,6-Difluorobenzoic Acid & 5.05 & 5.14 & 5.28 & 5.21 & 5.18 & 5.17 & 4.99 \\
\hline 3,4-Difluorobenzoic Acid & 5.12 & 5.08 & 5.06 & 5.10 & 5.07 & 5.03 & 4.99 \\
\hline 3,5-Difluorobenzoic Acid & 5.18 & 5.10 & 5.08 & 5.10 & 5.06 & 5.13 & 5.11 \\
\hline 2,3,4-Trifluorobenzoic Acid & 4.98 & 5.01 & 5.02 & 5.13 & 4.96 & 5.10 & 5.11 \\
\hline 2,3,6-Trifluorobenzoic Acid & 5.05 & 5.15 & 4.97 & 5.02 & 4.94 & 4.99 & 4.53 \\
\hline 2,4,5-Trifluorobenzoic Acid & 5.99 & 5.97 & 5.96 & 6.04 & 6.12 & 6.11 & 6.09 \\
\hline 2,4,6-Trifluorobenzoic Acid & 6.01 & 6.03 & $*$ & 6.01 & 5.68 & 5.67 & 5.62 \\
\hline 3,4,5-Trifluorobenzoic Acid & 5.18 & 5.14 & 5.15 & 5.12 & 5.10 & 5.18 & 5.25 \\
\hline 2,3,4,5-Tetrafluorobenzoic Acid & 5.74 & 5.74 & 5.76 & 5.71 & 5.76 & 5.70 & 5.68 \\
\hline 2,3,5,6-Tetrafluorobenzoic Acid & 6.29 & 6.30 & 6.40 & 6.36 & 6.19 & 6.26 & 6.29 \\
\hline Pentafluorobenzoic Acid & 5.59 & 5.64 & 5.73 & 5.64 & 5.68 & 5.69 & 5.69 \\
\hline$\alpha, \alpha, \alpha$-Trifluoro-o-toluic acid & 5.04 & 5.13 & 5.07 & 5.17 & 5.07 & 5.21 & 5.04 \\
\hline$\alpha, \alpha, \alpha$-Trifluoro-m-toluic acid & 5.08 & 5.15 & 5.13 & 5.19 & 5.05 & 5.22 & 5.19 \\
\hline$\alpha, \alpha, \alpha$-Trifluoro-p-toluic acid & 5.25 & 5.30 & 5.25 & 5.34 & 5.19 & 5.31 & 5.34 \\
\hline
\end{tabular}

*2,4,6-Trifluorobenzoic acid could not be quantitated at $\mathrm{T}=5$ due to the presence of an interference in the chromatogram. 
Table 4 Concentrations of Tracers in the Dark Tuff Samples

\begin{tabular}{llllllll}
\multicolumn{1}{c}{ Compound } & \multicolumn{7}{c}{ Concentration (ppm) } \\
& 0 & 1 & 5 & 10 & 20 & 30 & 60 \\
2,3-Difluorobenzoic Acid & 5.26 & 5.23 & 5.10 & 5.12 & 5.13 & 5.10 & 5.15 \\
2,4-Difluorobenzoic Acid & 5.38 & 5.44 & 5.35 & 5.36 & 5.29 & 5.41 & 5.47 \\
2,5-Difluorobenzoic Acid & 5.17 & 5.20 & 5.16 & 5.18 & 5.16 & 5.25 & 5.25 \\
2,6-Difluorobenzoic Acid & 5.07 & 5.22 & 5.40 & 5.41 & 5.50 & 5.73 & 5.04 \\
3,4-Difluorobenzoic Acid & 5.07 & 5.09 & 5.09 & 5.10 & 5.09 & 5.06 & 4.98 \\
3,5-Difluorobenzoic Acid & 5.06 & 5.22 & 5.01 & 5.04 & 5.02 & 5.13 & 5.12 \\
2,3,4-Trifluorobenzoic Acid & 4.96 & 5.06 & 5.05 & 5.14 & 5.05 & 5.18 & 5.12 \\
2,3,6-Trifluorobenzoic Acid & 5.07 & 5.13 & 4.93 & 4.99 & 4.99 & 5.03 & 5.02 \\
2,4,5-Trifluorobenzoic Acid & 5.95 & 5.97 & 5.96 & 5.99 & 6.12 & 6.14 & 6.11 \\
2,4,6-Trifluorobenzoic Acid & 5.85 & 5.92 & $*$ & $* 7.00$ & 5.67 & 5.65 & 5.70 \\
3,4,5-Trifluorobenzoic Acid & 5.07 & 5.10 & 5.08 & 5.16 & 5.20 & 5.13 & 5.39 \\
2,3,4,5-Tetrafluorobenzoic Acid & 5.74 & 5.76 & 5.80 & 5.68 & 5.79 & 5.72 & 5.62 \\
2,3,5,6-Tetrafluorobenzoic Acid & 6.29 & 6.33 & 6.22 & 6.13 & 6.18 & 6.23 & 6.32 \\
Pentafluorobenzoic Acid & 5.58 & 5.63 & 5.69 & 5.60 & 5.62 & 5.66 & 5.62 \\
$\alpha, \alpha, \alpha$-Trifluoro-o-toluic acid & 5.00 & 5.08 & 5.04 & 5.04 & 5.02 & 5.04 & 5.09 \\
$\alpha, \alpha, \alpha$-Trifluoro-m-toluic acid & 5.11 & 5.14 & 5.09 & 5.10 & 5.04 & 5.25 & 5.02 \\
$\alpha, \alpha, \alpha$-Trifluoro-p-toluic acid & 5.24 & 5.31 & 5.25 & 5.27 & 5.09 & 5.28 & 5.25
\end{tabular}

$* 2,4,6$-Trifluorobenzoic acid could not be quantitated at $\mathrm{T}=5$ due to the presence of an interference in the chromatogram. The high concentration reported on $\mathrm{T}=10$ is also due to an interference.

\section{COLUMN TESTING}

Another method used to measure the sorption of the tracer compounds to tuff material is the column test. The tracer is injected into a column containing ground tuff and the time required for its elution is measured. The elution volume, calculated by multiplying the elution time by the measured flow rate, is compared to that of bromide. Bromide, which is considered to be a conservative tracer, is used as a reference for each compound. All fluorinated benzoates have been tested on columns containing each of the tuffs (see the previous report for column dimensions and detection methods). The mean elution volume, the standard deviation, and the percent relative standard deviation (\%RSD) for each compound are listed in Tables 5 - 7. Two or three injections of potassium bromide were made per day and the ratios of the elution volumes, analyte/bromide, were calculated. This ratio is also listed along with the mean, standard deviation (SD), and percent relative standard deviation (\%RSD) for the $\mathrm{KBr}$ elution volumes.

The elution volumes for all acids are very similar to bromide and are generally within one standard deviation. The ratios, Tracer/KBr, are also very close to one, and in fact in the light and medium tuff most are less than one. This indicates faster travel through the column than bromide. The Tracer/KBr in the dark tuff are slightly higher than in the light and medium tuff, but, the elution volumes for $\mathrm{KBr}$ injections are still within one standard deviation of the benzoates. If it is assumed that bromide (potassium bromide) does not sorb to the tuff then all the benzoic and toluic acids also act conservatively. 
Table 5 Column Test Results for the Medium Tuff

Compound

2,3-difluorobenzoic acid

2,4-difluorobenzoic acid

2,5-difluorobenzoic acid

2,6-difluorobenzoic acid

3,4-difluorobenzoic acid

2,3,4-trifluorobenzoic acid

2,3,6-trifluorobenzoic acid

2,4,5-trifluorobenzoic acid

2,4,6-trifluorobenzoic acid

3,4,5-trifluorobenzoic acid

2,3,4,5-tetrafluorobenzoic acid

2,3,5,6-tetrafluorobenzoic acid

pentafluorobenzoic acid

m-toluic acid

o-toluic acid

p-toluic acid

$\begin{array}{lc}\text { Mean } & \text { Standard } \\ (\mathrm{ml}) & \begin{array}{c}\text { Deviation } \\ 206.6\end{array} \\ 162.6 & 4.77 \\ 158.2 & 7.74 \\ 161.0 & 5.81 \\ 209.3 & 5.48 \\ 156.4 & 1.65 \\ 206.6 & 3.74 \\ 201.4 & 1.67 \\ 158.3 & 9.81 \\ 210.8 & 2.02 \\ 191.6 & 7.83 \\ 213.0 & 1.44 \\ 207.4 & 1.26 \\ 210.4 & 3.34 \\ 205.7 & 1.20 \\ 212.5 & 2.42\end{array}$

Tracer
\%RSD
0.97
2.93
4.89
3.61
2.62
1.05
1.81
0.83
6.20
0.96
4.09
0.68
0.61
1.59
0.58
1.14

Tracer/

Kbr

0.99

0.96

0.98

1.00

0.98

0.99

0.99

0.98

0.98

1.00

0.99

1.00

1.00

0.97

0.98

1.01
$\mathrm{KBr} \mathrm{KBr}$

Mean SD

208.10 .82

$169.5 \quad 2.52$

$161.5 \quad 2.64$

$160.6 \quad 2.53$

212.94 .05

$157.5 \quad 6.18$

$208.6 \quad 3.08$

$205.5 \quad 1.60$

$161.5 \quad 3.73$

$211.7 \quad 2.34$

$193.7 \quad 0.00$

$212.4 \quad 2.38$

$206.5 \quad 1.41$

$215.9 \quad 4.45$

$209.7 \quad 0.52$

$210.5 \quad 0.60$
$\mathrm{KBr}$

$\%$ RSD

0.39

1.49

1.64

1.58

1.90

3.92

1.48

0.78

2.31

1.11

0.00

1.12

0.68

2.06

0.25

0.29

Table 6 Column Test Results for the Light Tuff

Compound

2,3-difluorobenzoic acid

2,4-difluorobenzoic acid

2,5-difluorobenzoic acid

2,6-difluorobenzoic acid

3,4-difluorobenzoic acid

3,5-difluorobenzoic acid

2,3,4-trifluorobenzoic acid

2,3,6-trifluorobenzoic acid

2,4,5-trifluorobenzoic acid

2,4,6-trifluorobenzoic acid

3,4,5-trifluorobenzoic acid

2,3,4,5-tetrafluorobenzoic acid

2,3,5,6-tetrafluorobenzoic acid pentafluorobenzoic acid

m-toluic acid

o-toluic acid

p-toluic acid

$\begin{array}{lcr}\begin{array}{l}\text { Mean } \\ \text { (ml) }\end{array} & \begin{array}{c}\text { Standard } \\ \text { Deviation }\end{array} & \begin{array}{r}\text { Tracer } \\ \text { \%RSD }\end{array} \\ 301.7 & 6.16 & 2.04 \\ 288.4 & 13.0 & 4.51 \\ 292.7 & 5.34 & 1.82 \\ 287.0 & 10.2 & 3.56 \\ 292.6 & 14.7 & 5.04 \\ 302.5 & 5.13 & 1.70 \\ 292.7 & 7.31 & 2.50 \\ 292.9 & 5.54 & 1.89 \\ 282.0 & 9.07 & 3.21 \\ 287.1 & 13.9 & 4.84 \\ 292.8 & 3.77 & 1.29 \\ 292.1 & 13.9 & 4.77 \\ 292.4 & 10.3 & 3.51 \\ 292.6 & 28.3 & 9.67 \\ 294.3 & 12.3 & 4.19 \\ 287.4 & 11.9 & 4.14 \\ 285.5 & 13.9 & 4.87\end{array}$

Tracer/

$\mathrm{KBr}$

$\mathrm{KBr} \quad \mathrm{KBr} \quad \mathrm{KBr}$

mean SD \%RSD

$\begin{array}{llll}0.95 & 316.4 & 7.91 & 2.50\end{array}$

$\begin{array}{llll}0.92 & 312.0 & 21.3 & 6.84\end{array}$

$\begin{array}{llll}0.99 & 296.7 & 3.30 & 1.11\end{array}$

$\begin{array}{llll}0.95 & 302.3 & 3.74 & 1.24\end{array}$

$\begin{array}{llll}0.98 & 298.2 & 12.7 & 4.26\end{array}$

$\begin{array}{llll}0.95 & 317.8 & 11.4 & 3.59\end{array}$

$\begin{array}{llll}0.95 & 309.5 & 7.02 & 2.27\end{array}$

$\begin{array}{llll}0.94 & 312.9 & 14.1 & 4.51\end{array}$

$\begin{array}{llll}0.92 & 305.1 & 3.65 & 1.20\end{array}$

$\begin{array}{llll}0.95 & 302.8 & 3.64 & 1.20\end{array}$

$\begin{array}{llll}0.95 & 308.0 & 3.76 & 1.22\end{array}$

$\begin{array}{llll}0.93 & 315.2 & 12.4 & 3.95\end{array}$

$\begin{array}{llll}0.92 & 316.3 & 18.0 & 5.70\end{array}$

$\begin{array}{llll}0.98 & 299.1 & 19.9 & 6.66\end{array}$

$\begin{array}{llll}0.96 & 306.0 & 25.6 & 8.36\end{array}$

$\begin{array}{llll}0.93 & 309.2 & 3.85 & 1.25\end{array}$

$\begin{array}{llll}0.90 & 316.1 & 16.8 & 5.30\end{array}$ 
Table 7 Column Test Results for the Dark Tuff

Compound

2,3-difluorobenzoic acid

2,4-difluorobenzoic acid

2,5-difluorobenzoic acid

2,6-difluorobenzoic acid

3,4-difluorobenzoic acid

3,5-difluorobenzoic acid

2,3,4-trifluorobenzoic acid

2,3,6-trifluorobenzoic acid

2,4,5-trifluorobenzoic acid

2,4,6-trifluorobenzoic acid

3,4,5-trifluorobenzoic acid

2,3,4,5-tetrafluorobenzoic acid

2,3,5,6-tetrafluorobenzoic acid

pentafluorobenzoic acid

m-toluic acid

o-toluic acid

p-toluic acid

$\begin{array}{lc}\begin{array}{l}\text { Mean } \\ (\mathrm{ml})\end{array} & \begin{array}{c}\text { Standard } \\ \text { Deviation }\end{array} \\ 177.4 & 7.29 \\ 269.9 & 6.55 \\ 237.6 & 20.2 \\ 152.8 & 4.68 \\ 159.1 & 0.56 \\ 245.1 & 10.3 \\ 250.2 & 9.55 \\ 152.8 & 4.68 \\ 163.0 & 6.05 \\ 135.2 & 3.49 \\ 169.4 & 1.51 \\ 162.2 & 2.51 \\ 170.9 & 1.07 \\ 241.9 & 10.3 \\ 243.1 & 2.96 \\ 138.4 & 3.19 \\ 165.5 & 9.18\end{array}$

$\begin{array}{lll}\mathrm{KBr} & \mathrm{KBr} & \mathrm{KBr} \\ \text { mean } & \mathrm{SD} & \% \mathrm{RSD} \\ 171.4 & 2.49 & 1.46 \\ 266.5 & 9.96 & 3.73 \\ 242.1 & 28.9 & 11.9 \\ 152.8 & 3.79 & 2.48 \\ 155.4 & 0.85 & 0.55 \\ 240.2 & 6.74 & 2.81 \\ 240.0 & 14.0 & 5.82 \\ 152.8 & 3.79 & 2.48 \\ 162.2 & 10.5 & 6.47 \\ 140.5 & 4.05 & 2.88 \\ 168.0 & 0.21 & 0.12 \\ 164.7 & 3.43 & 2.08 \\ 169.7 & 2.23 & 1.31 \\ 230.2 & 9.72 & 4.22 \\ 230.4 & 4.65 & 2.02 \\ 141.4 & 5.96 & 4.22 \\ 167.0 & 13.1 & 7.85\end{array}$

\section{METHOD DEVELOPMENT}

At this time, the degree of dilution that will occur during the tracer test is largely unknown. A dilution of at least 6 orders of magnitude is expected due to the large distance and high pumping rate involved with these tests. It was therefore recommended by HRC that a preliminary tracer test be performed This will allow all aspects of the test to be examined and further optimized prior to the larger more costly testing.

It is the current plan to inject 2,6-difluorobenzoic acid. Method development to optimize the analysis of this compound is therefore in progress. We are currently investigating the effects of injecting larger sample volumes into the HPLC. We also plan to develop concentrating procedures using solid phase extraction. 


\section{2,3-Difluorobenzoic Acid}

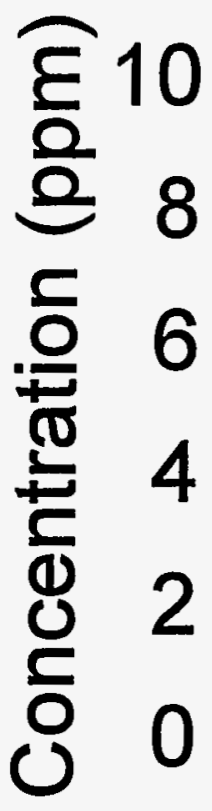

0

10

20

30

50

60

Time (days)

- Light Tuff Tuff $\rightarrow$ Med Tuff $\quad \bullet$ Dark Tuff

$\rightarrow$ Light Tuff (dup) $-z$ Dark Tuff (dup) $\rightarrow$ - Control 


\section{2,5-Difluorobenzoic Acid}

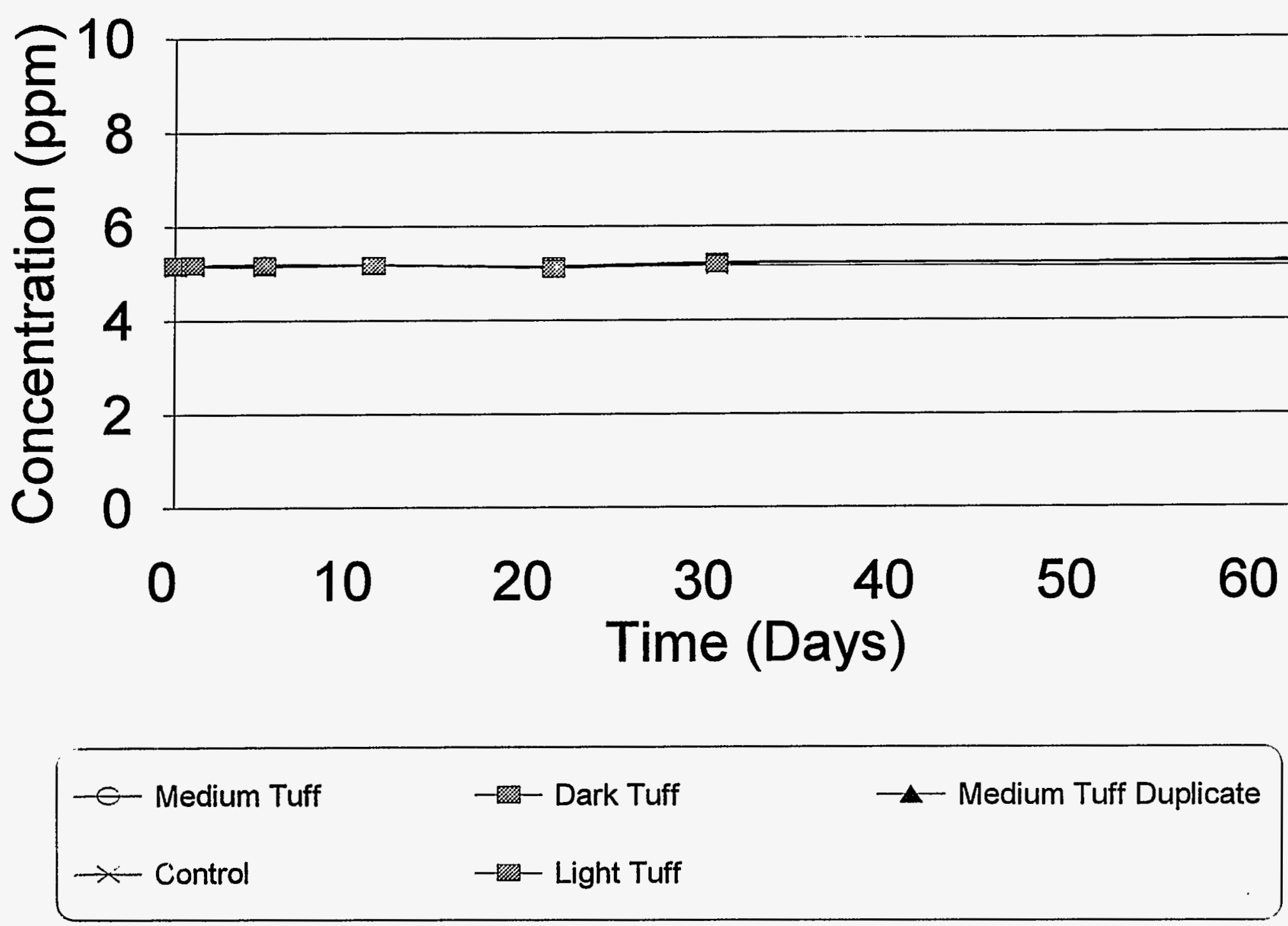


Figure 3

\section{2,6-Difluorobenzoic Acid}

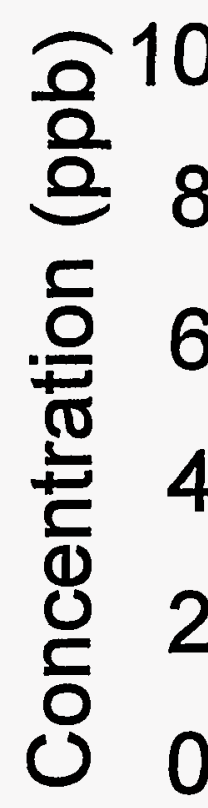

$\begin{array}{llllllllll}0 & 10 & 20 & 30 & 40 & 50 & 60 & 70 & 80 & 90\end{array}$ Time (days)

$\rightarrow$ - Light Tuff $\rightarrow$ Med Tuff $\rightarrow-$ Dark Tuff $-\Delta$ - Control 


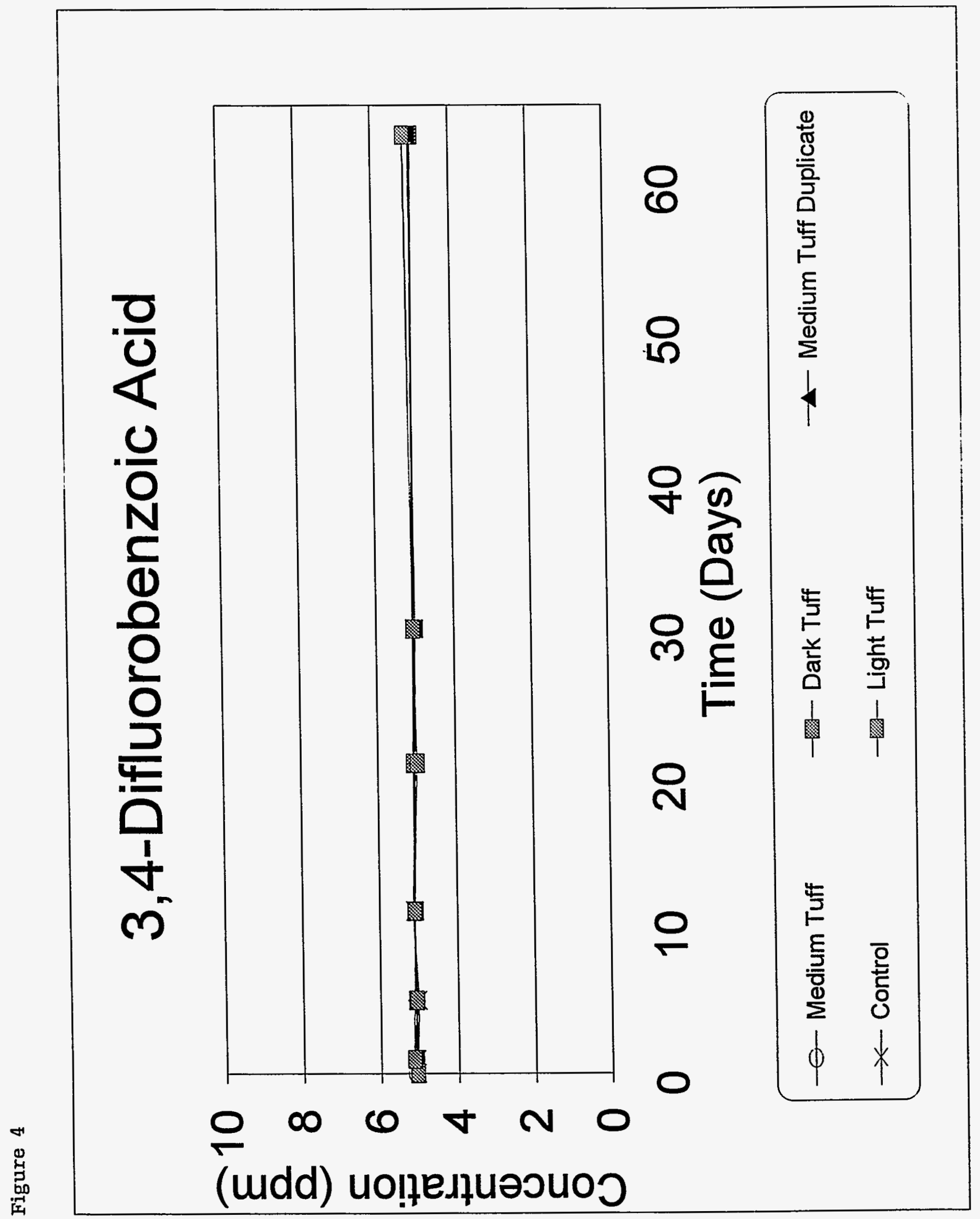




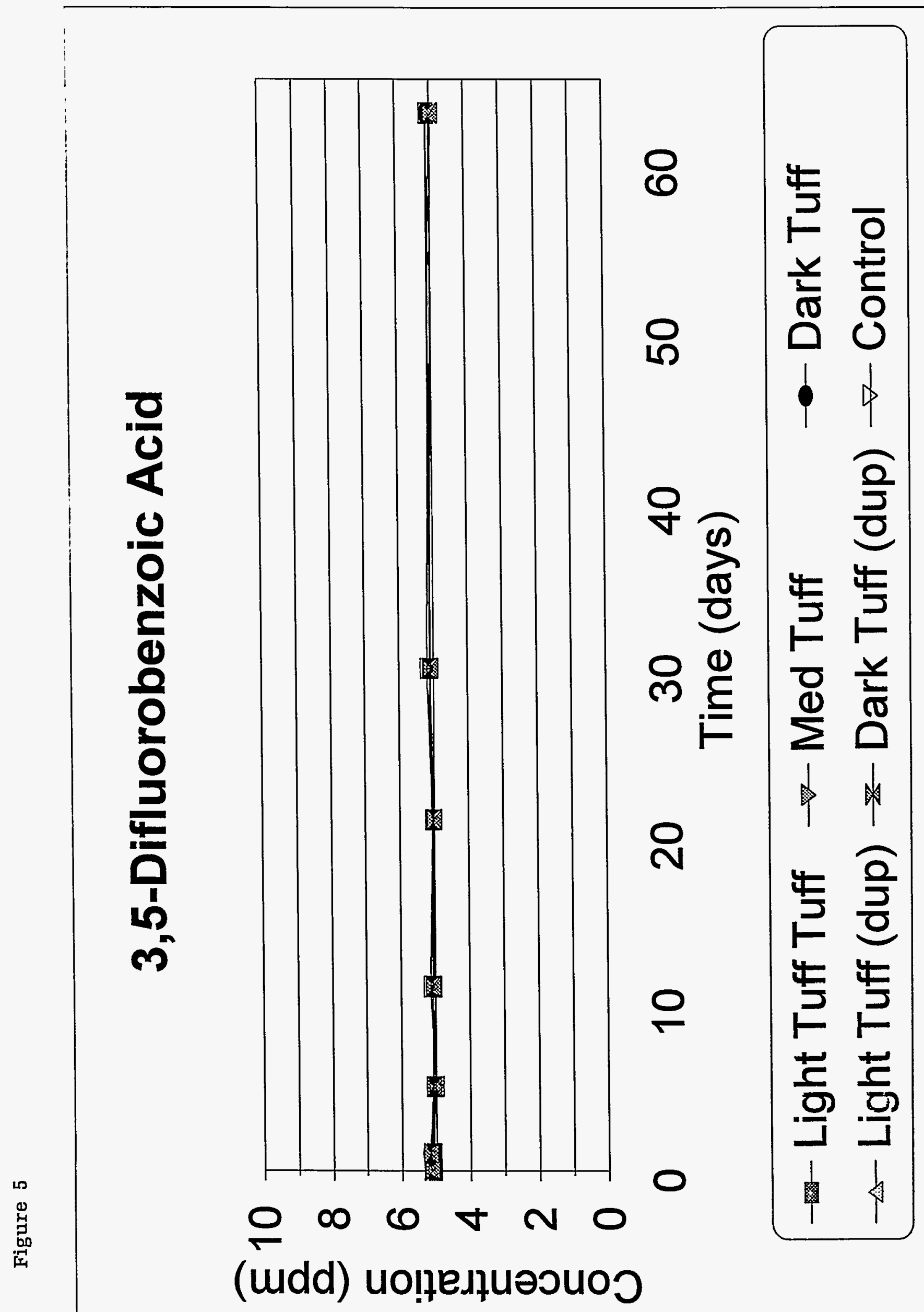




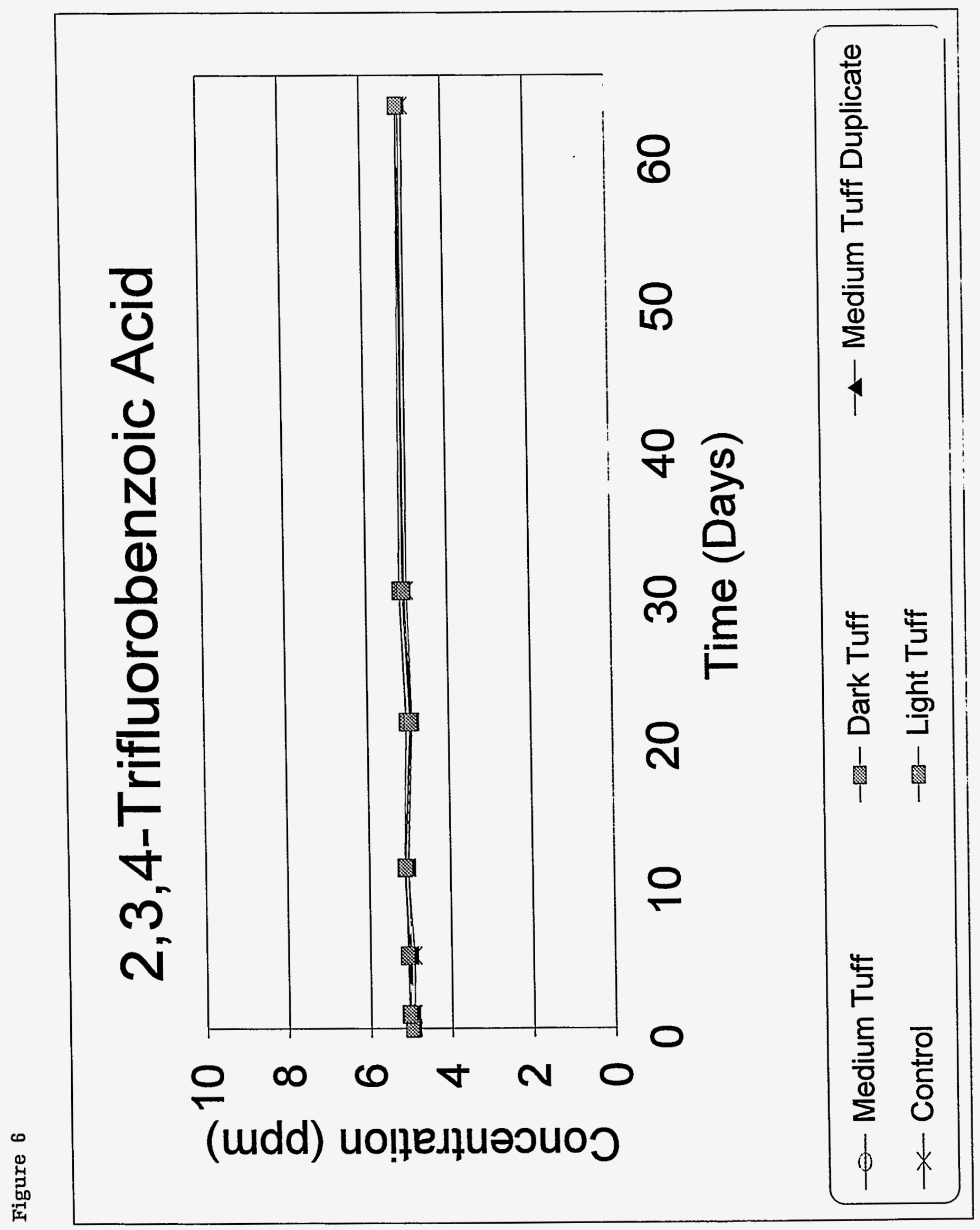




\section{2,3,6-Trifluorobenzoic Acid}

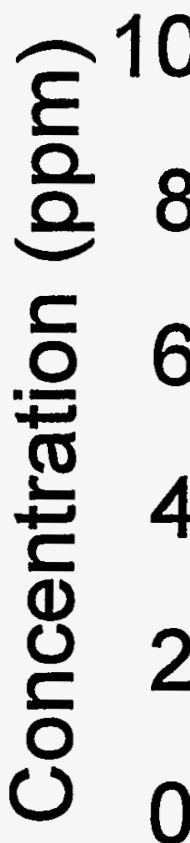
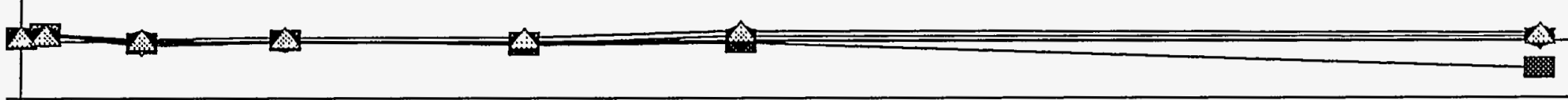

0

0

10

20

30

40

50

60

Time (days)

$\begin{array}{ll}\rightarrow-\text { Med Tuff } & \rightarrow \text { Dark Tuff } \\ \rightarrow-\text { Dark Tuff (dup) } & - \text { Control }\end{array}$




\section{2,4,5-Trifluorobenzoic Acid}

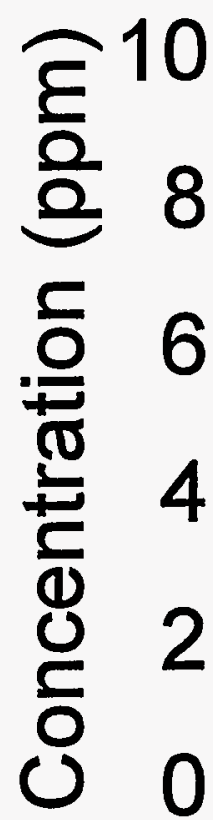

0

10

20

Time (Days)

$\leftarrow$ Medium Tuff

-Dark Tuff

$\neg$ Medium Tuff Duplicate

- Control

- - Light Tuff 
Figure 9

\section{2,4,6-Trifluorobenzoic Acid}

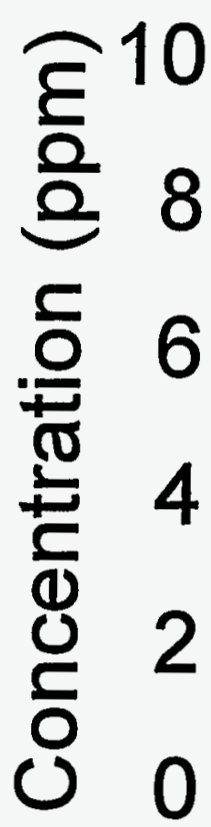

0

10

20

30

40

50

60

Time (Days)

- - Medium Tuff

- - Dark Tuff

$\rightarrow-$ Medium Tuff Duplicate - - Control 


\section{3,4,5-Trifluorobenzoic Acid}

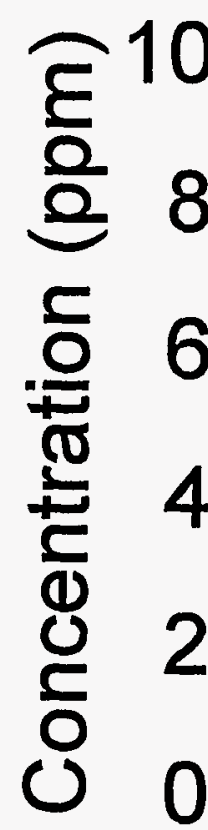

0

10

20

30

40

50

60

Time (days)

- Light Tuff Tuff $\rightarrow$ Med Tuff $\quad \bullet$ Dark Tuff

$\triangle$ Light Tuff (dup) $\rightarrow$ - Dark Tuff (dup) $\rightarrow$ Control 


\section{2,3,4,5-Tetrafluorobenzoic Acid}

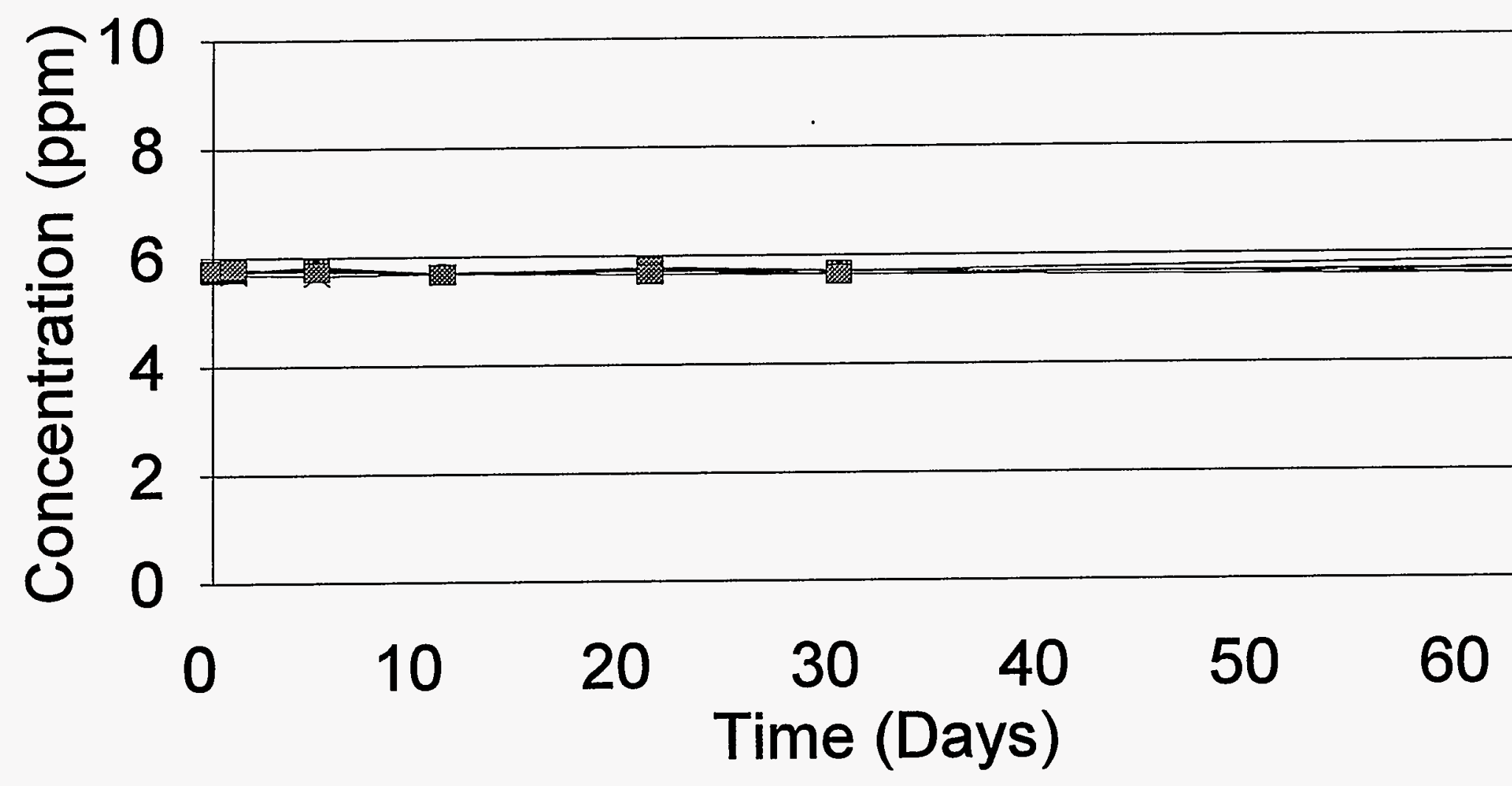

$\begin{array}{ll}-\in \text { Medium Tuff } & - \text { - Dark Tuff } \\ -\leftarrow \text { Control } & - \text { - Light Tuff }\end{array}$




\section{2,3,5,6-Tetrafluorobenzoic Acid}

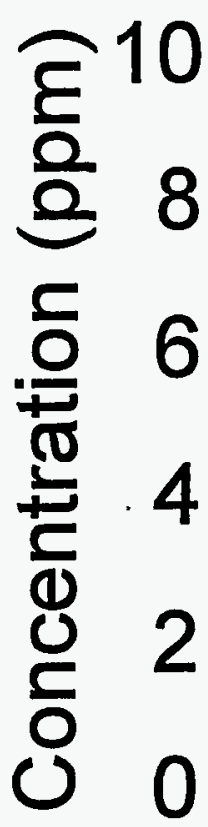

0

10

20

30

40

50

60

Time (Days)

$$
\begin{array}{ll}
-\epsilon-\text { Medium Tuff } & \triangle \text { Dark Tuff } \\
-\varpi-\text { Medium Tuff Duplicate } & \leftarrow \text { Control }
\end{array}
$$




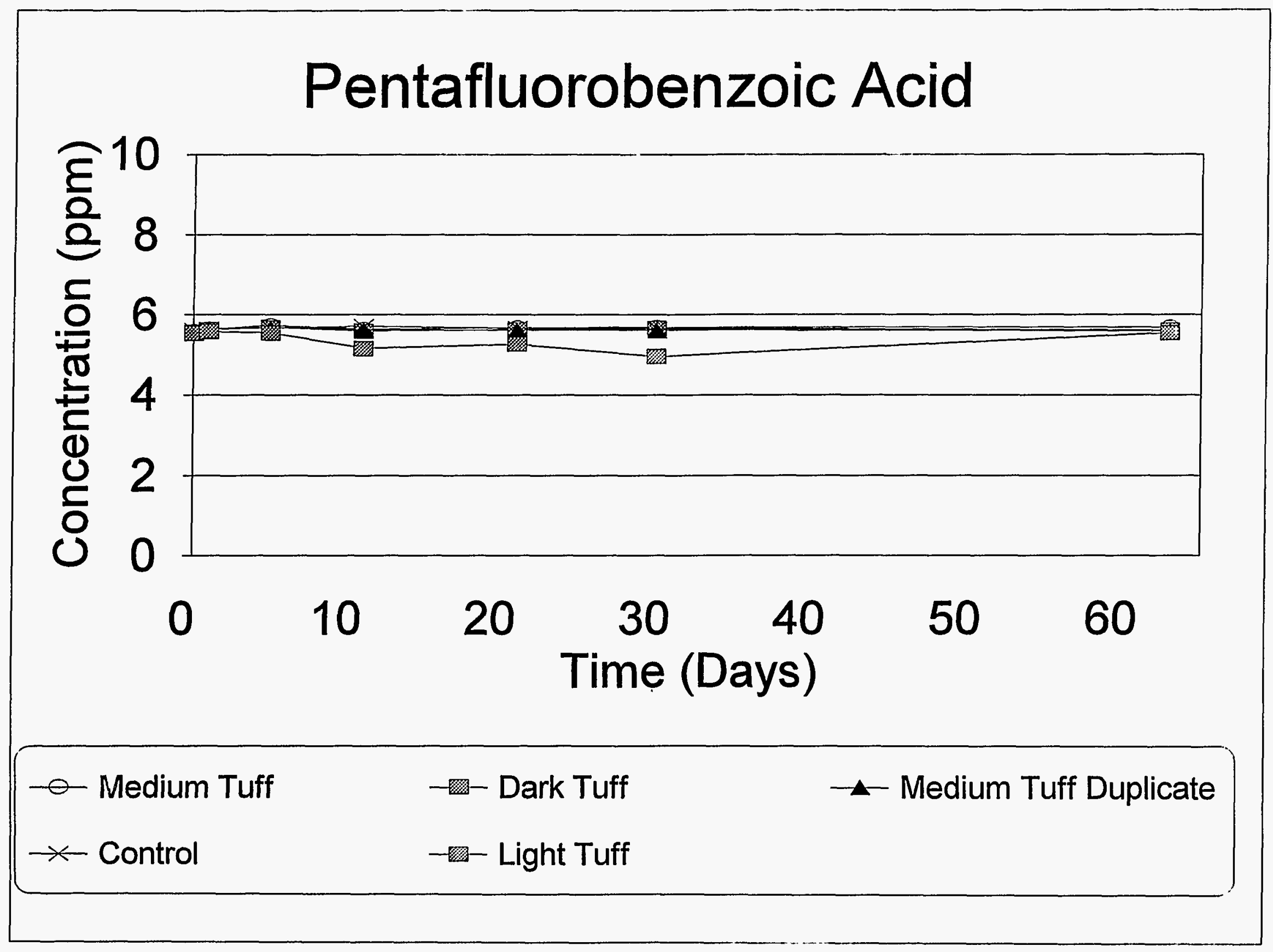




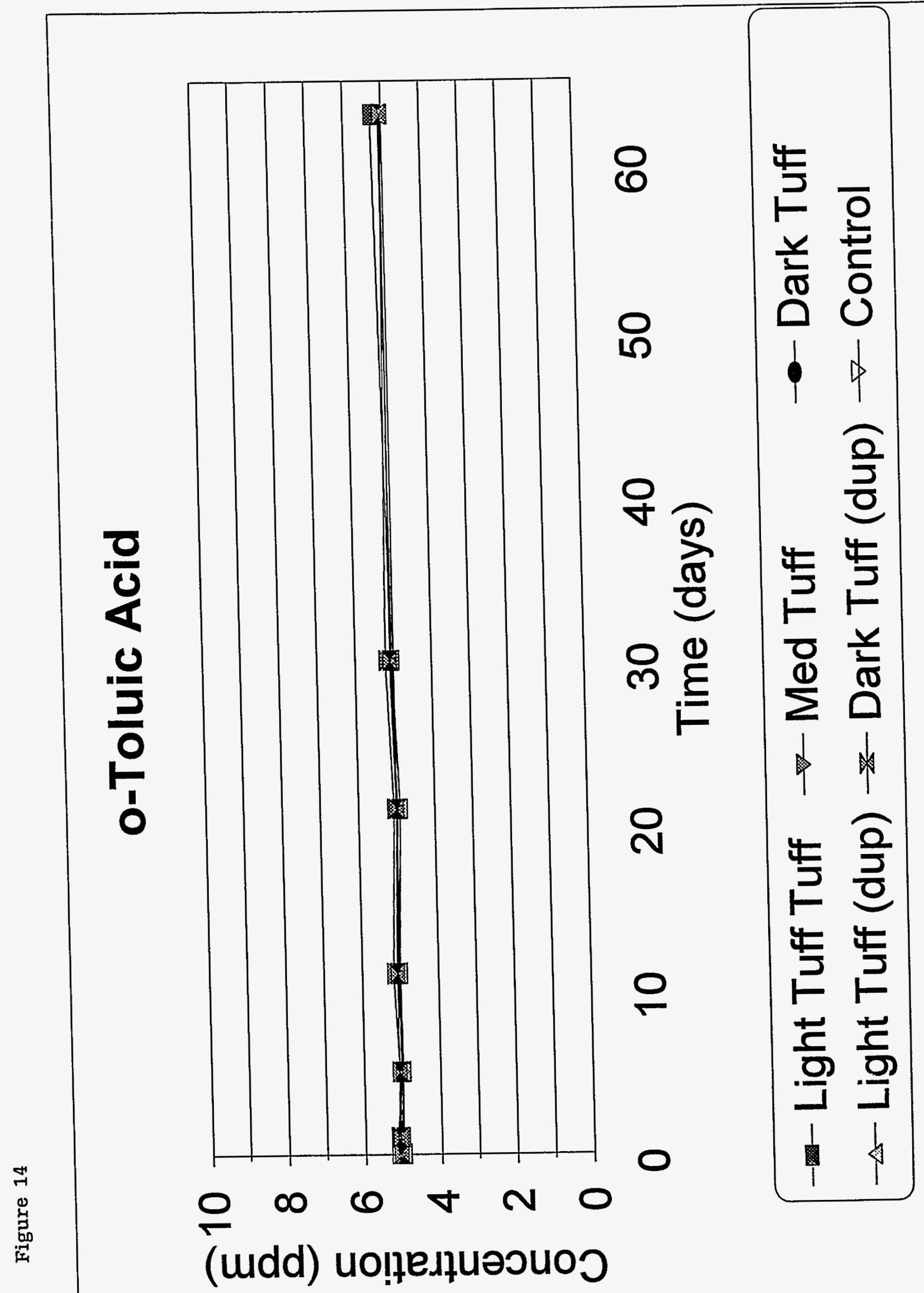




\section{m-Toluic Acid}

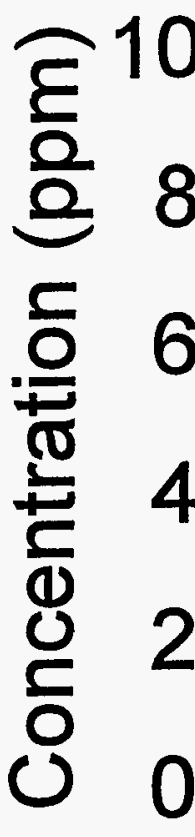

0

10

20

30

40

50

60

Time (days)

$\rightarrow$ Light Tuff Tuff $\rightarrow$ Med Tuff $\quad \bullet$ Dark Tuff

$-\Delta$ Light Tuff (dup) $\rightarrow$ Dark Tuff (dup) $\rightarrow$ Control 


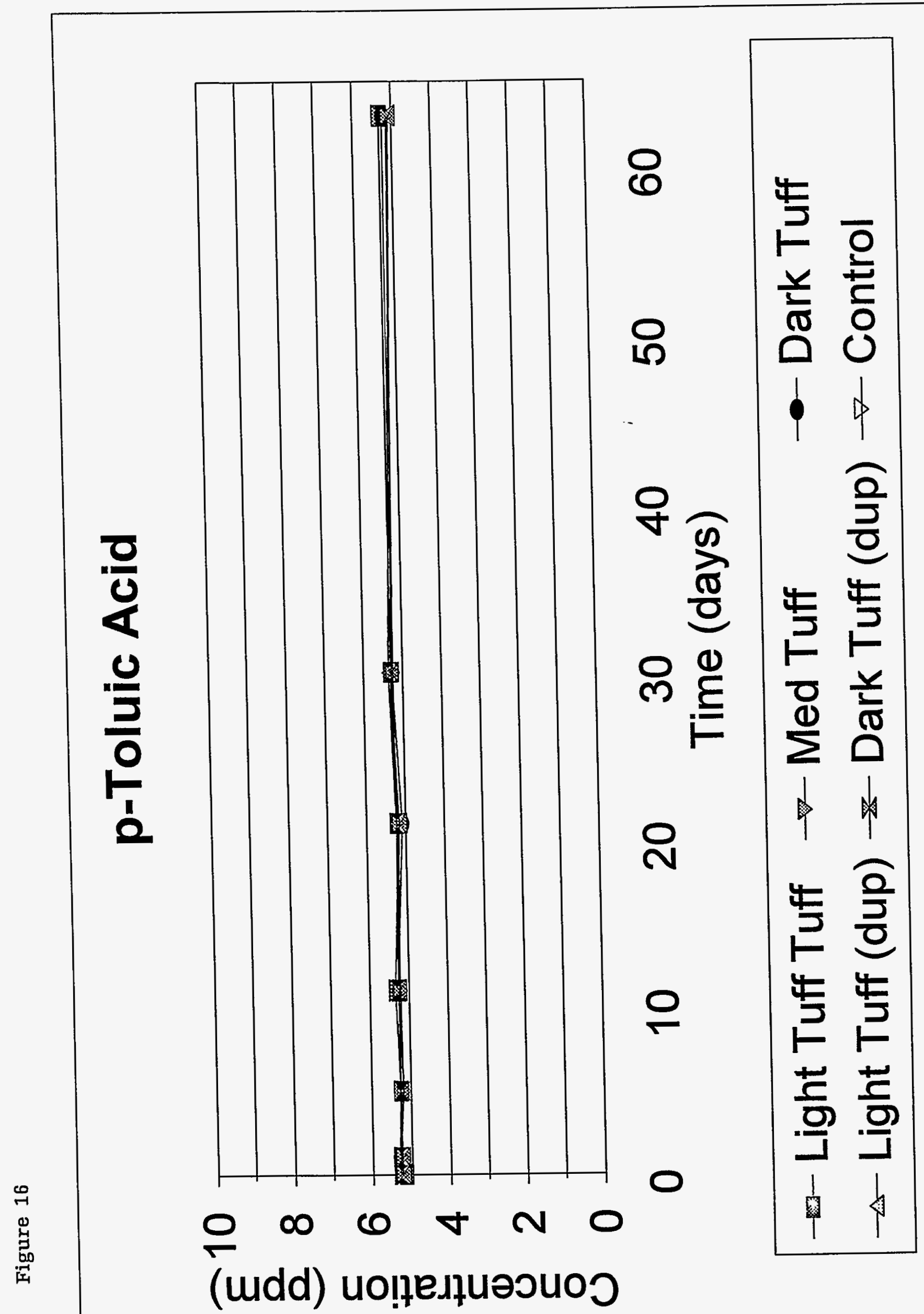




\section{2,4-Difluorobenzoic Acid}

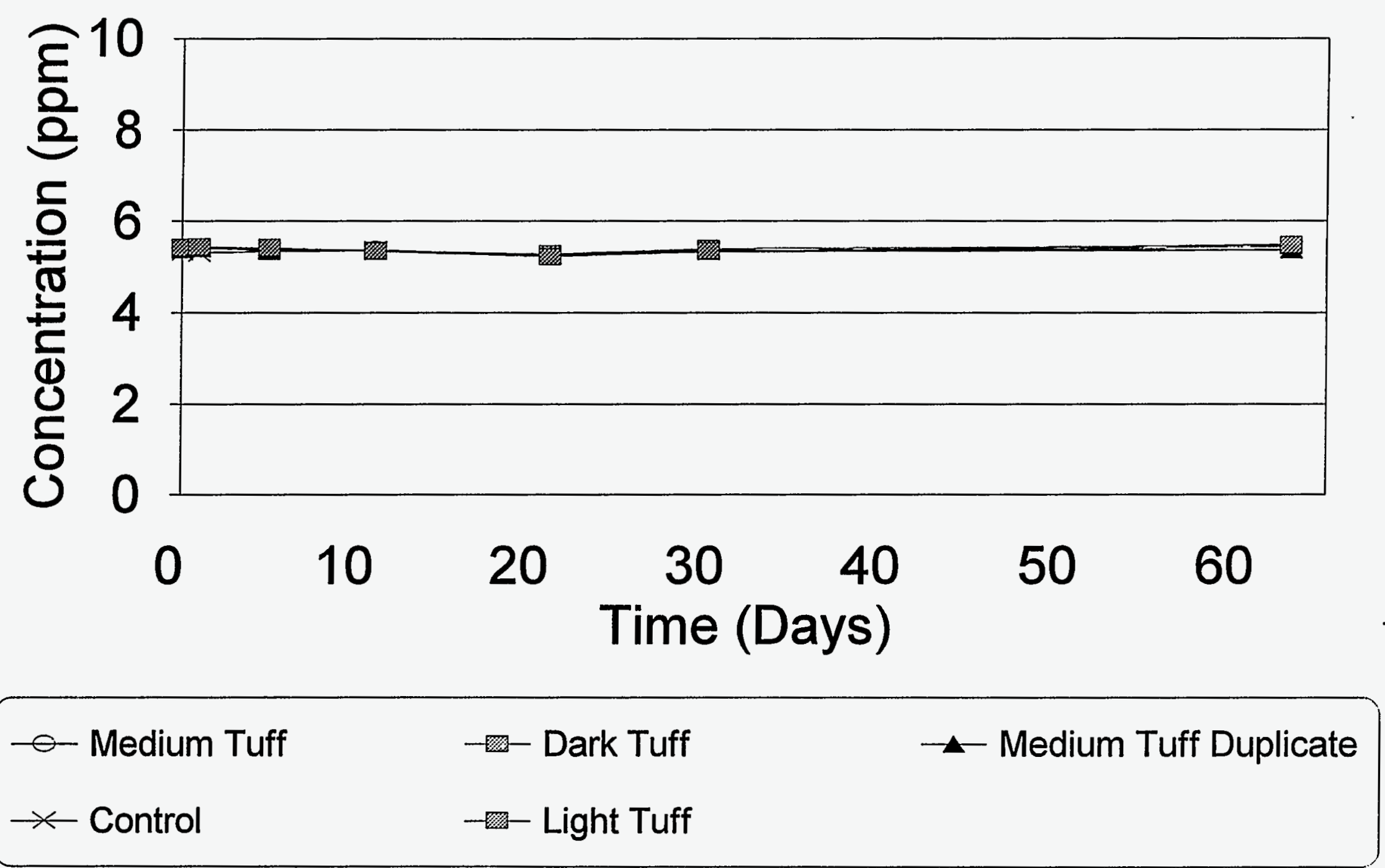

